\title{
Formación docente en un espacio virtual de aprendizaje: Una experiencia concreta en el contexto Chileno.
}

\author{
Juán Silva \\ jsilva@comenius.usach.cl \\ Centro Comenius \\ Universidad de Santiago de Chile
}

\section{Introducción.}

El curso a distancia en la modalidad b-learning para la actualización docente "Geometría.cl: Aprender Geometría Creando Soluciones", es una iniciativa con cobertura en todo el territorio Chileno y fue financiado por el Ministerio de Educación de Chile a través del Centro de Perfeccionamiento, Experimentación e Investigaciones Pedagógicas (CPEIP). Ha sido desarrollado por el Centro para el desarrollo de innovaciones en educación de la Universidad de Santiago de Chile. La formación se dirigió a docentes del $5^{\circ}$ a $8^{\circ}$ grado de enseñanza primaria, en contenidos relacionados a la enseñanza de la geometría, se inserta en el marco de la reforma curricular, e incorpora recursos TIC en las actividades de aprendizaje.

El curso nace en el contexto de una línea de formación docente con apoyo de componente virtual implementada por el CPEIP, este es el cuarto curso desarrollado. Por otra parte un estudio reciente realizado en el marco del proyecto Enlaces muestra que el $92 \%$ de los establecimientos cuenta con infraestructura tecnológica y el 76\% de los docentes han sido capacitados en el uso de TIC, lo anterior como resultado de la implementación del proyecto Enlaces. Por otra parte la penetración en el uso de TIC en el profesorado es creciente un $80 \%$ de los docentes con equipos en los hogares, $51 \%$ de ellos con Internet, 58\% de ellos con banda ancha (Collect y Enlaces 2004).

El desarrollo e implementación de la experiencia contempla: a) la selección y formación de tutores, b) el diseño pedagógico del curso, c) el diseño e implementación del curso en la plataforma Moodle; d) desarrollo de diversos recursos de apoyo a los contenidos, e) aplicación de Pre y Post Test y evaluaciones sumativas y formativas.

El curso capacitó a nivel nacional 786 docentes, divididos en 29 cursos, con un promedio de 27 alumnos por curso. Para el apoyo tutorial durante la implementación del curso se generó una comunidad de tutores para apoyarles en sus tareas de tutorización del curso en las áreas: administrativas, técnicas, social y pedagógica. La metodología de trabajo situó al docente en el centro del aprendizaje, como una aprendiz que define en forma autónoma su camino de aprendizaje. En este contexto el participante construye conocimiento a través de la interacción con: los materiales, el tutor y los compañeros.

Este artículo presenta los aspectos más relevantes de esta experiencia: la selección y formación de tutores, el diseño pedagógico del curso y su implementación en la plataforma y los principales resultados de su ejecución. En este último aspecto se tiene que 786 docentes rindieron la primera evaluación del curso, 645 rindieron la prueba final, lo cual refleja un 82\% de retención. Un promedio de 670 participantes equivalente al $85 \%$ de los participantes se conectaron semanalmente al curso, el cual lo aprueban 598 un 92\% de los que lo finalizan. El balance es altamente positivo, el cual se manifiesta en los avances en los aprendizajes que se reflejan en diferencias positivas entre el PreTest y PostTest, una alta valoración de los participantes respecto a los contenidos y actividades, los recursos propuestos, las estrategias de enseñanza, la metodología de trabajo implementada, así como del uso de la plataforma y el rol del tutor.

Las TIC están produciendo cambios en la formas de enseñanza y aprendizaje, en la forma en que los 
profesores y aprendices se relacionan con el conocimiento y las forma en que los agentes involucrados en el proceso educativo interactúan. El uso de las TIC en educación ha permitido relevar propuestas metodológicas que pedagógicamente llevan años de desarrollo (Gros, 2002). Uno de estos aspectos es el constructivismo y especialmente el constructivismo social. Desde la perspectiva sociocultural del aprendizaje, la interacción social y el discurso, son elementos básicos para el desarrollo de los procesos cognitivos superiores (Vygotsky, 1978). Como señala Hernández (1997) las publicaciones de revistas de tecnología educativa son de las que más páginas han dedicado al tema del constructivismo.

El impacto de las TIC en la comunicación es tal que se denomina Comunicación Mediada por Computador a todas estas instancias de comunicación sincronas o asincronas realizadas por medios de recursos tecnológicos. Dentro de las herramientas comunicativas se encuentra la llamada Conferencia Mediada por Computador (CMC), una modalidad asincrónica de comunicación que permite la comunicación utilizando como base el texto escrito (Ryan et.al., 2000; Salmon, 2000; Harasim et.al., 2000; Bates, 1995). En los últimos años ha crecido rápidamente el CMC, especialmente en su modalidad asíncrona (Tolmie y Boyle, 2000). Esta herramienta comunicativa incorporada de modo apropiado en los procesos de enseñanza o formación, y bien asistidas pueden favorecer la colaboración "un proceso interactivo y colectivo de producción de conocimiento en donde los alumnos producen el conocimiento activamente formulando las ideas por escrito que son compartidas y construidas a partir de las reacciones y respuestas de los demás" (Harasim et.al. 2000, p.24). La CMC ofrece enormes posibilidades para crear entornos de aprendizaje basados en modelos constructivista de carácter sociocultural, posibilitando el trabajo colaborativo y potenciando la construcción de conocimiento en una comunidad de aprendizaje. Se promueve espacios para la reflexión, accesible a toda hora, adaptables al ritmo de aprendizaje individual y por sobre todo opuestos a la clásica transmisión de conocimiento.

Las experiencias educativas que utilicen estos entornos virtuales de aprendizaje (EVA), tanto si se ubican en escenarios de enseñanza a distancia, presencial o mixta, requieren una redefinición de los elementos organizativos del aprendizaje, en relación a: los agentes involucrados (profesores, participantes, administrativos); los espacios donde se lleva a cabo las actividades formativos (casa, centro educativo, aulas informáticas, lugar de trabajo); los tiempos; y secuencias de aprendizajes (Pérez, 2002).

Para el correcto funcionamiento de una CMC, que facilite la interacción social y la construcción de conocimiento, en forma colaborativa al interior de una comunidad de aprendizaje, se requiere un tutor "profesor virtual" que modere la conferencia. En relación al rol del tutor en los EVA se plantean nuevas competencias y habilidades: deben estar preparado para mantener "vivos" los espacios comunicativos, facilitar el acceso a los contenidos, generar un dialogo efectivo con los participantes y entre los participantes, de modo que se favorezca el aprendizaje activo, la construcción de conocimiento cooperativo y/o colaborativo. Estos profesionales son fundamentales en le éxito de las experiencias de enseñanza y formación que utilizan CMC para la colaboración (Cabero, 2001; Salmon, 2000; Ryan et.al., 2000).

Para Swan y otros colaboradores que investigaron los factores de éxito de los ambientes de aprendizajes online asincrónicos, hay tres factores que contribuyen significativamente, estos son: una interface transparente, un instructor que interactúe frecuentemente y constructivamente con los estudiantes, y una discusión valorada y dinámica (Swan et al., 2000). Para quiénes analizan las interacciones no tansolo desde el punto de vista cuantitativo, sino cualitativo, la frecuencia y calidad de las intervenciones en una CMC estará en gran medida marcada por las actividades moderadoras que efectúe el tutor (Perez, 2002).

Existe consenso que la actualización docente, es una de las claves para la implementación de procesos de enseñanza innovadores, que potencien más y mejores aprendizajes. Las TIC pueden apoyar los procesos de formación continua de los docentes a través de los espacios virtuales de aprendizaje, creando instancias formativas donde la interacción, la colaboración y el aprendizaje en compañía de los pares y apoyados por un tutor, se transformen en ricos escenarios para la actualización docente. El que los docentes usen estos espacios virtuales para formarse, les permite conocer de forma más directa los roles que se esperan de un "profesor virtual”, el que ellos podrían ejercer al incorporar esta modalidad de enseñanza en sus prácticas docentes.

Las experiencias de formación virtual de docentes, permite a estos profesionales acceder a actualizaciones en línea, diseñadas normalmente por equipos pedagógicos calificados y de cobertura a nivel nacional o 
internacional, además de la adquisición de parte de los profesores de nuevas competencias y habilidades para enfrentar en mejores condiciones una próxima experiencia en esta área. Este tipo de formación podría transformarse, en el futuro, en una de las principales fuentes de formación y actualización docente. Diversos países en distintos continentes están invirtiendo en esta modalidad. Sin embargo es necesario tener presente que las características específicas de la profesión docente, evitándose la utilización acrítica de modelos de otras industrias y profesiones (Grünberg , 2002). Empieza a haber experiencias interesantes en el que se muestra cómo el uso de las tecnologías puede facilitar enormemente el trabajo de los profesores y su propia formación. La creación de comunidades virtuales para compartir recursos y conocimientos así como la creación de espacios de práctica compartidos son una muestra (Gros y Silva 2005).

\section{Objetivos}

Objetivo General: Diseñar e implementar un curso virtual para apoyar la adquisición de conocimientos y metodologías relacionados a la geometría en el segundo ciclo de enseñanza primaria, favoreciendola interacción al interior de una comunidad de aprendizaje.

Objetivos Específicos: Proveer a los participantes de actividades y recursos de aprendizaje para adquirir nuevos conocimientos y/o reforzar los existentes; Poner a disposición de los docentes un conjunto de recursos y estrategias metodológicas para favorecer la enseñanza de la geometría; Mostrar logros en los aprendizaje de los profesores y profesoras participantes; Favorecer las interacciones entre los participantes del curso al interior de una comunidad de aprendizaje

\section{Metodología}

El desarrollo e implementación de la experiencia contempló:

- la selección y formación de tutores, para lo cual se uso el modelo e-modetaring de Salmon, creándose actividades como objetos de aprendizaje. Se diseño un perfil para seleccionar a los tutores y se les formo mediante un curso en la modalidad e-learning que concluyó con un encuentro presencial.

- diseño pedagógico del curso, el cual ha sido concebido bajo un modelo interactivo para la enseñanza de la matemática cuya concepción se acerca mucho a la expresión del Madison Project, que se sintetiza en: “conjetura - trata, pon la idea a prueba - observa lo que sucede y... aprende cómo seguir”;

- diseño e implementación del curso en la plataforma Moodle, contempló la organización de los contenidos en unidades, las cuales poseen tres áreas: Actividades y Evaluación: se encuentra con el conjunto de actividades organizadas semanalmente, dentro de la semana por día y al interior del día, las actividades específicas con una breve descripción y tiempo estimado de desarrollo, considera una evaluación formativa semanal y una sumativa por unidad; Interacciones: contempla un foro de discusión, un espacio para consultas y un diario mural; Biblioteca: agrupa los diferentes recursos como lecturas, guías, Applets, material de referencia.

- Desarrollo de diversos recursos de apoyo a los contenidos: guías, material de referencia, applets, lecturas, referencias a sitios, ente otros recursos.

- La aplicación de un Pre y Post Test: Se aplicó al inicio del curso un pre test y al final un post test.

- Obtención y análisis de la información como: datos estadísticos de participación en presenciales, evaluaciones sumativas en la plataforma y registro de participaciones en espacios interactivos en la plataforma.

\section{Selección y formación de tutores}

Las habilidades que posee un docente en un ámbito presencial aún siendo un excelente docente no garantizan el éxito cuando se actúa entornos virtuales, es necesaria una formación del tutor para dotarlo de las habilidades necesarias para cumplir adecuadamente en los aspectos sociales, pedagógicos, técnicos y administrativos, especialmente los dos primeros. 
Los participantes se seleccionaron según un perfil que el tutor debería cumplir para desempeñarse adecuadamente en las labores propias que la tutorización que un curso virtual demanda. Se contó con 60 postulantes, $55 \%$ quedaron en la categoría seleccionados, $13 \%$ en seleccionados con reserva y $32 \%$ rechazados. De los seleccionados 42 participaron finalmente del curso de tutores, de ellos 22 un 52\% tiene experiencia previa como tutores en cursos online.

Para la capacitación se diseñó y desarrolló un curso virtual que tuvo como propósito proveer los conocimientos y herramientas que se requieren para apoyar y facilitar el proceso educativo de terceros a través de entornos virtuales de aprendizaje (EVA). La formación consideró los siguientes contenidos: la plataforma del curso y sus distintos recursos; aspectos característicos básicos de la formación a distancia y su situación actual; el rol del tutor en los EVA; técnicas pedagógicas, sociales y administrativasen un curso a distancia utilizando TIC y medios telemáticos; contenidos, metodología y recursos del "Curso de geometría”.
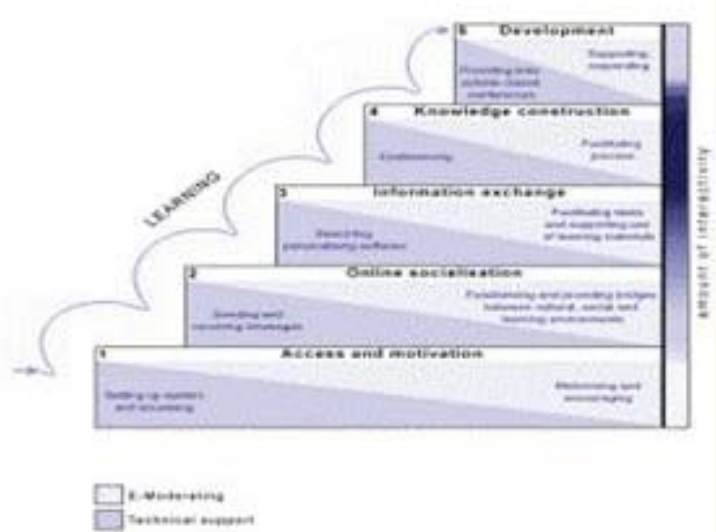

Figura 1: Modelo E-moderating
Se utilizó el modelo E-Moderating (Figura 1) ampliamente reconocido en la investigación que se basa en una investigación acción la profesora Gilly Salmon de la Open University (Salmon 2000).

En cada Unidad, se desarrollarón actividades, las que se diseñaron como objetos de aprendizaje conformado por : Una descripción, objetivo, la tara a desarrollar, los recursos a utilizar, los tiempos estimados, fecha de inicio y termino y la evaluación formativa o sumativa

La capacitación formal se realizó durante 4 semanas, y contempló 60 horas a distancia y 6 horas presenciales. La estrategia de aprendizaje buscó que los alumnos -futuros tutores- vivenciarán el modelo de formación a distancia que se espera implementen posteriormente cuando autoricen su curso, se les propuso situaciones tipos y se les pidió explicitar formas de actuar. Todo lo anterior en el marco de una propuesta de trabajo que favorezca la construcción de conocimiento en un contexto social, donde la reflexión, la discusión con los pares, la puesta en común de las ideas, y el debate sean elementos centrales.

La evaluación del curso consideró actividades de tipo formativas y sumativas. Se evaluó sobre la base de la realización de un conjunto de actividades propuestas para cada unidad, más participación en los espacios virtuales prestando principal atención al tipo de intervenciones de los tutores. Esto último porque se espera que los futuros tutores vayan realizando intervenciones tipos en cada una de las etapas del modelo descrito anteriormente.

La calificación final se calculó ponderando el promedio aritmético de la primer unidad al $40 \%$ y el promedio de la segunda unidad al 60\%. El promedio general alcanzo a un 6,5 - la nota final en la escala de 1 a 7 - Siendo el promedio en la Unidad 1 un 6,7 y en la unidad II un 6,3. El 86\%, 36 alumnos aprobaron el curso. De los 42 participantes que iniciaron el curso un 14\% (6) desertan y un 86\%(36) finalizan el proceso de formación de tutores.

Con los tutores que se desempeñaron finalmente en el curso se diseñó una comunidad virtual para acompañarles en su tarea tutorial, resolviendo dudas, conociendo las nuevas unidades y potenciandoel actuar del tutor a través de documentos, noticias y debates. El crear estas comunidades es un aspecto considerado por autores como Harasim et. al (2000), como relevante para apoyar a los tutores especialmente cuando éstos no están relacionados con la generación de los contenidos como ocurre en este curso. 
El curso Geometría.cl es una invitación a los profesores para que descubran por si mismos lo fascinante que puede ser el adentrarse en las profundidades de un conocimiento que tiene mucha historia y del cual estamos totalmente rodeados en nuestra vida cotidiana, una invitación a disfrutar y maravillarsecon la construcción hecha, la hipótesis comprobada, la relación descubierta, el desafío resuelto. En otras palabras: aprender geometría creando soluciones.

En relación a la práctica docente, el curso tiene como propósito que los profesores y profesoras participantes: obtengan conocimientos acerca de los contenidos geométricos que deban enseñar a sus estudiantes; tengan acceso a herramientas metodológicas y didácticas que les faciliten lograr aprendizajes pertinentes y de calidad con sus estudiantes; adquieran conocimientos y competencias para el uso de recursos tecnológicos en el contexto de su trabajo de aula. En relación a las interacciones el propósito es discutir sobre temática relacionadas a la enseñanza y aprendizaje de la geometría, así como de la práctica docente y compartir recursos para apoyar su enseñanza y experiencias relacionas a buenas prácticas docentes.

\section{Estructura del curso y Contenidos}

El curso se estructura en cuatro unidades temáticas a distancia y tres sesiones presenciales. De las cuatro unidades temáticas, una corresponde al uso de la plataforma donde se desarrolla el curso- Moodle- y las otras tres corresponden a los contenidos del curso: Formas geométricas en el plano, formas geométricas en el espacio y transformaciones en el plano. Las presenciales son tres y de ellas la última es obligatoria. La Primera sesión presencial: Conocimiento del tutor y los compañeros, aplicación de Pretest, exploración de la plataforma, presentación de la primera unidad y su evaluación la Segunda sesión presencial: análisis de la experiencia y aprendizajes logrados en la unidad I, aspectos problemáticos o dudas respecto a la plataforma presentación de las unidades 2 y 3 y su evaluación; la Tercera sesión presencial. Evaluación general de la experiencia y aplicación del Post-Test.

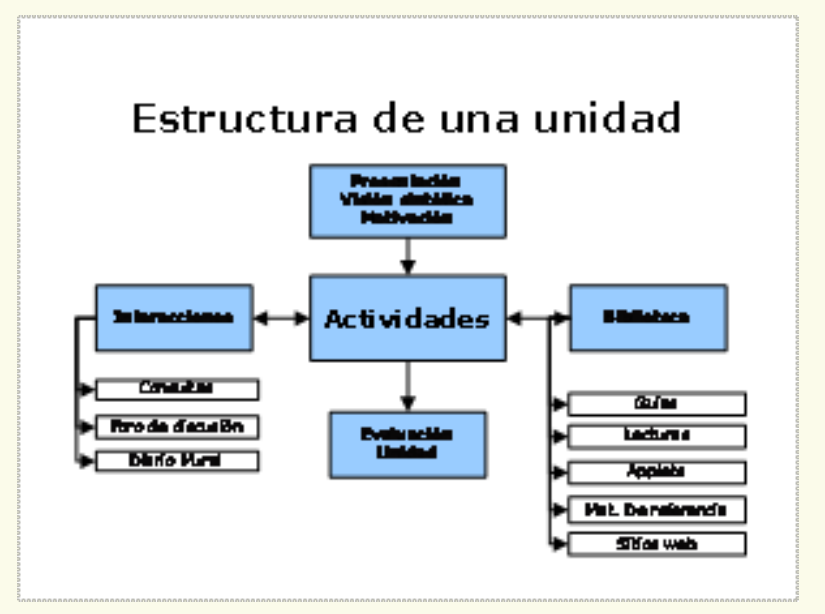

Figura 2: Estructura de una unidad
Las unidades temáticas (Figura 2) esta compuesta de una visión, interacciones, un conjunto de actividades y evaluaciones y recursos en la biblioteca.

Las actividades se organizaron en semanas y días, calculando que la actividad diaria fuese posible en una hora y media de trabajo. El tiempo de dedicación requerido para tener éxito en este curso es de una hora y media diarias [1] , considerando jornadas de Lunes a Viernes, lo que da un total de siete horas y media ( 7.5 horas) semanales.

Las actividades consistieron en: lecturas, desarrollo de guías, visitas a sitios web, búsquedas en la web, visualizaciones (geometría mental), construcciones geométricas, trabajo con figuras de papel, construcciones de cuerpos en cartulina, cálculos, demostraciones (unas pocas) y trabajo con el computador, en particular mediante applets y un procesador geométrico.

\section{Materiales}

Los docentes participantes en el curso, además de interactuar con los contenidos en la Web del curso recibieron material que les permitiera volver a repasar los contenidos trabajados y utilizarlos en la enseñanza con sus alumnos. En efecto ellos recibieron los siguientes materiales, en forma impresa: Portafolio - Recurso para contener el material impreso entregado en el curso- documento Descripción del curso; Manual de Moodle para el participante; Los materiales de referencias de las unidades I, II y III. En forma digital: Un CD con los recursos de las Unidades I, II y III contiene guías (83), lecturas (16), applests (60), material de referencia (3), software (4), presentaciones(2).

\section{EI modelo de aprendizaje}


El modelo de aprendizaje se basa en una concepción constructiva del conocimiento, en la que los participantes y los docentes del curso, tutores y equipo pedagógico, constituyen una comunidad de aprendizaje.

La concepción del conocimiento y del aprendizaje matemático del curso, se fundamenta en el "modelo interactivo para el aprendizaje matemático" generado en el marco del proyecto FONDEF “Aprender matemática creando soluciones” del Centro Comenius/USACH. La visión más sintética que podemos ofrecer de la forma de pensar y de actuar que proponemos, se acerca mucho a la expresión del Madison Project: “conjetura - trata, pon la idea a prueba - observa lo que sucede y... aprende cómo seguir”.

Las actividades del curso se organizan siguiendo la secuencia: exploración, búsqueda de regularidades y conjeturas; formalización, puesta en común y sistematización; cierre, práctica, aplicaciones y dominio de la técnica. Esto se acompaña con actividades interactivas en las cuales los participante tienen la oportunidad de realizar consultas relacionados con los contenidos -foro consulta-, compartir recursos Diario Mural- y debatir respecto a temáticas relacionadas -foro de discusión-.

\section{El curso en la plataforma}

El curso en la plataforma se estructura con diversos elementos que buscan dar vida al modelo pedagógico del curso el cual se sitúa en la epistemología constructivista, en el cual el participante es constructor de su propio conocimiento. Esta construcción se logra en la apuesta del curso a través de dos vías una de carácter individual y otra de carácter social. En el primer aspecto se pone a disposición de los estudiantes diversos materiales relacionados con los contenidos del curso, en el segundo se hace uso de diversas herramientas asíncronas para lograr interacción entre los participantes y entre éstos y el tutor.

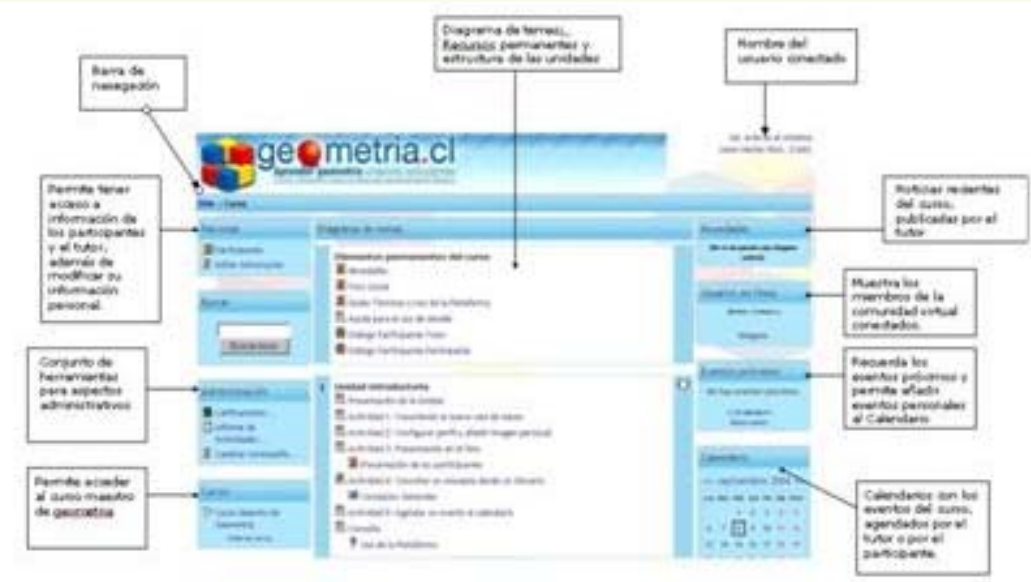

La figura describe los diferentes elementos con los que se encuentra el participante en el curso como diagrama del curso; notificación de novedades; eventos próximos, calendario, herramientas de administración, entre otros.

Figura 3: Interface del curso en la plataforma

\section{Elementos Permanentes}

Los elementos permanentes son espacios transversales a los contenidos de las unidades temáticas del curso y están disponibles durante toda la ejecución del curso, en el se encuentran los foros Novedades: destinado a mantener informados a los participantes de los aspectos relevantes del curso desde el punto de vista pedagógico y administrativo; Foro Social: espacio para la interacción en temas planteados por los participantes y no relacionados con los contenidos; Dudas técnicas y uso de la plataforma: espacio para realizar consultas respecto al uso de la plataforma; las páginas web: Ayuda para el uso de Moodle: ayuda en formato web para el uso de la plataforma al modo de preguntas frecuentes y geometria.cl: descripción del curso; los diálogos entre los participantes y entre estos y el tutor.

\section{Las unidades}

Las unidades (Figura 4) organizan el trabajo de 4 semanas considera los aspectos de contenidos geométrricos, interacción y evaluación.

Ha sido segmentado en cuatro áreas: a) presentación donde 


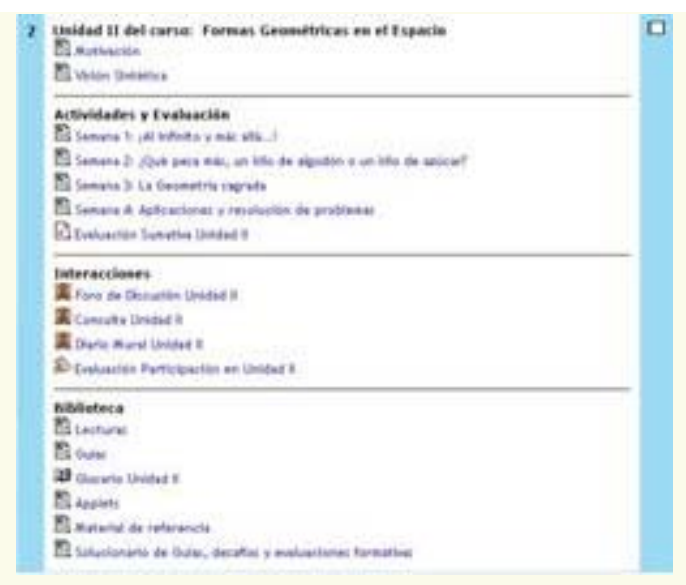

esta la Motivación y la visión sintetica; b) Actividades y Evaluación: contiene las actividades de la unidad organizada pos semanas y el cuestionario correspondiente a la Evaluación sumativa de la unidad; c) Interacciones: contiene los espacios para el intercambio foro de discusión, diario mural y consultas; d) Biblioteca: espacio que contiene los recursos del curso organizado por tipo de recursos: Guías, lecturas, material de referencia, Applets, Glosario, solucionarios de guías y desafíos.

Figura 4: Estructura de una unidad

Para una mejor comprensión de esta forma de organizar las unidades a continuación se detallarán sus espacios centrales.

\section{Semana y días}

Como una forma de organizar el trabajo que se espera el participante realice durante el desarrollo del curso se organizaron los contenidos por semanas y dentro de ellas por día.

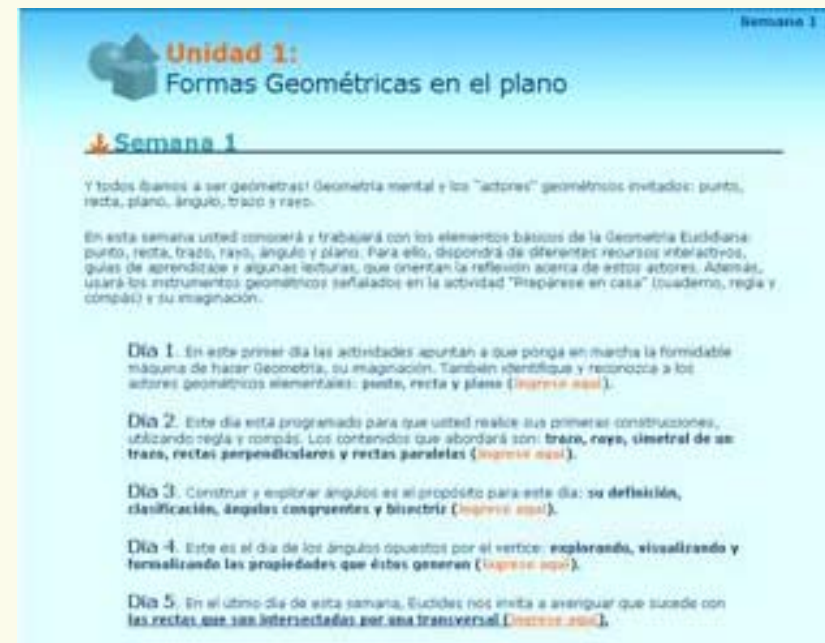

Figura 5: Estructura semana

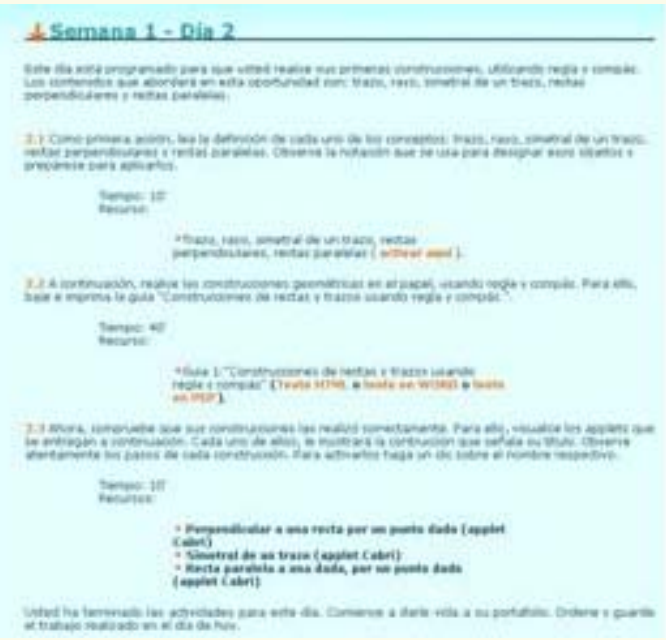

Figura 6: Estructura día

Una semana (Figura 5) aglutina un conjunto de actividades distribuidas por día, que comienza con una motivación e introducción al trabajo semanal, una descripción del trabajo diario, concluye con una evaluación formativa en el día 5 y un llamado a usar los espacios interactivos.

El día (Figura 6) presenta las actividades a realizar diriamente, las que se describen brevemente, indican el tiempo de ejecución estimado y el recurso a utilizar para su desarrollo. Es un organizador del trabajo diario, que sugiere una forma de trabajo, pero el participante posee la flexibilidad de reorganizar el día de acuerdo sus conocimientos previos, focalizando en los que domine menos.

Esta estructura de día y semana responde a lo planteado por Barberà y Badia(2004) en el sentido de presentar en forma clara la organización de las actividades y que se articulen a través de ellas el acceso a los diferentes recursos.

\section{Los Participantes}

Se matricularon en el curso 1.004 personas, de las cuales un $29 \%$ fueron hombres y el restante $71 \%$ mujeres. Respecto de la edad, la mayor parte de ellos tienen entre 45 a 54 años.

En relación a la dependencia de los establecimientos a los que pertenecen los participantes en el curso, un 
$54 \%$ pertenecen a colegios particulares subvencionados, un $44 \%$ pertenecen a colegios municipalizados, es decir un $98 \%$ pertenecen al sistema subvencionado -dependen directa o indirectamente del gobiernoEl costo del curso es de aproximadamente 17 Euros para profesores del sistema subvencionado, 76 Euros para profesores de colegios particulares.

Con respecto a la experiencia, un 53\% de los matriculados no ha tenido experiencia en cursos a distancia a través de Internet, mientras que el restante $47 \%$ si ha tenido. Los participantes se distribuyen en 29 cursos con un promedio de 35 alumnos por curso.

\section{Resultados}

En esta sección se presentan los principales resultados del curso, ellos se han obtenido a través de los diferentes sistemas de registro de información como: la aplicación del Pre y Post Test, la asistencias a las presenciales, los resultados de las evaluaciones sumativas en la plataforma y los datos obtenidos de la plataforma respecto a las participación en los espacios interactivos.

\section{Participación en el curso}

Durante todas las semanas se llevó un monitoreo de los alumnos activos en el curso, emitiéndose un reporte semanal el cual da cuenta de la cantidad de alumnos activos e inactivos en la semana, además de contabilizar los sin ninguna conexión en el curso. La figura 7 muestra el comportamiento de estos parámetros en las 14 semanas de ejecución del curso.

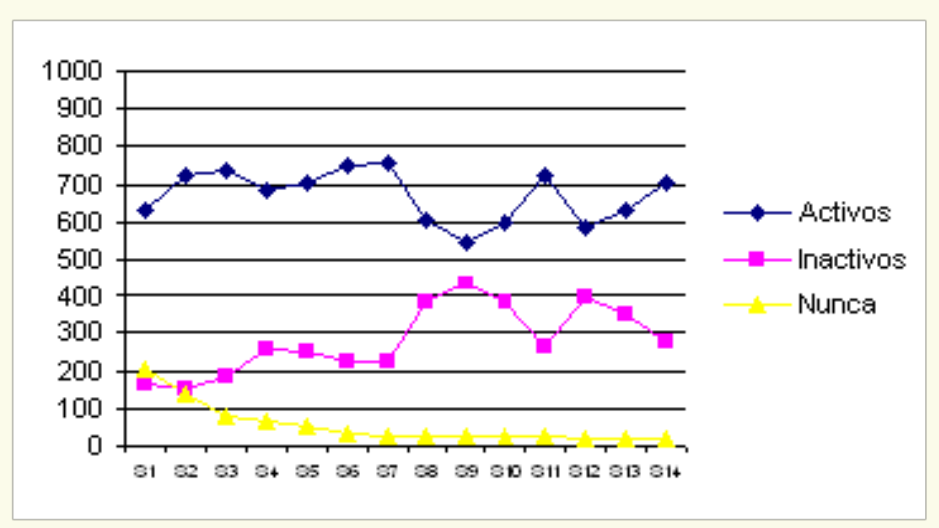

El promedio de alumnos conectados semanalmente alcanza a 670 un $85 \%$ de los 786 participantes. Han entrado alguna vez a la plataforma durante el desarrollo del curso 950 alumnos es decir un 94\% de los 1.004 inscritos.

Sólo dos semanas registran una cantidad de activos menor a 600, ellas coinciden con el término del año semana 9 y semana 12 .

Figura 7: Actividad semanal

\section{Participación Sesiones Presenciales}

El curso contempla tres presenciales, al inicio, termino del curso y después de la primera unidad de contenidos. Para el desarrollo de estas presenciales se entrego al tutor una planificación a seguir con las actividades a desarrollar y recursos digitales como presentación para su apoyo.

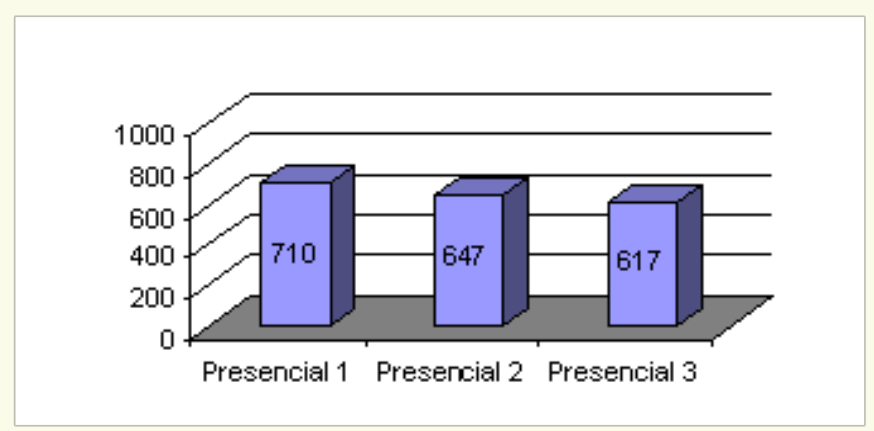

La figura 8 muestra la asistencia a las tres presenciales.

Se observa una diferencia entre la asistencia a la primera presencial y la última de 93 participantes lo que representa un $87 \%$ de asistencia a la presencial 3, respecto a la primera presencial.

Figura 8: Participación en presenciales

\section{Participación espacios de intercambio}

En esta sección se analizará la participación de los participantes en los diversos espacios asíncronos contemplados para la comunicación entre el tutor con los alumnos y entre los propios participantes. 


\section{Participación en los espacios permanentes}

Los espacios permanentes son un conjunto de herramientas principalmente foros que están disponibles para el uso por parte de los participantes a lo largo de todo el curso.

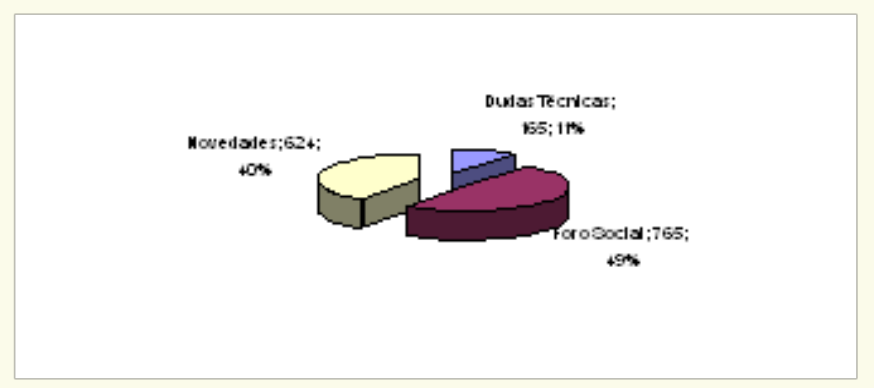

El foro social (Figura 9) concentra el 49\% de los temas abiertos por los participantes. Las noticias "Novedades" alcanzan al $40 \%$ y finalmente las “Dudas Técnicas” un $11 \%$.

Figura 9: Uso de elementos permanentes

Se presenta 165 dudas técnicas un promedio de 5.5 por curso. Estas dudas se relacionan al uso de la plataforma y la configuración de los computadores para correr ciertas aplicaciones como los Applets.

En el foro social hay 765 temas abiertos por los participantes, al interior de ellos hay diversos niveles de interacción difíciles de cuantificar, el promedio es de 26.3 temas abiertos por curso, recordar que estos temas los inician y animas los propios participantes, existiendo nula o escasa participación del tutor, salvo en el foro de bienvenida que inicia el tutor en este espacio. El foro social pasa a ser una especie de "sala de profesores virtual”.

En novedades espacio restringido a publicaciones sólo del tutor que no pueden ser debatidas por los participantes se registran 624 intervenciones con un promedio de 21.5 intervenciones. Estas corresponden a información y orientaciones que los tutores hacen llegar a sus alumnos respectos al desarrollo de las actividades, rendición de evaluaciones y criterios de evaluación, entre otras.

\section{Participación en espacios interactivos}

La participación en los espacios interactivos si bien es variable en cada unidad, sigue tendencias similares que posteriormente se reflejan en el global de las tres unidades, en este sentido el foro de discusión concentra la mayor parte de las intervenciones, seguido del foro diario mural y consultas.

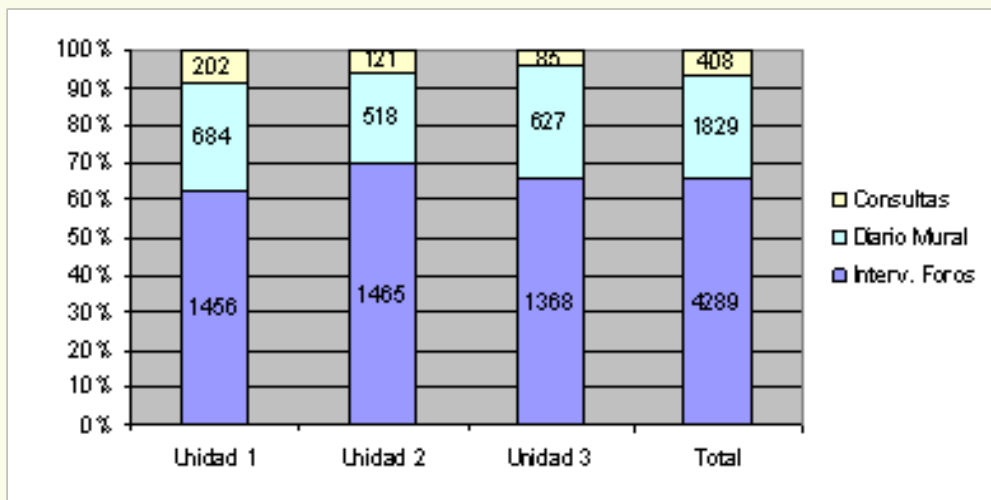

A nivel general se alcanza un $66 \%$ de intervenciones en los foros, $28 \%$, diario mural y $6 \%$ consultas

Se alcanza promedios por unidad en cada curso de: 50.3 intervenciones en el foro, 4.6 consultas y 21.6 aportes al diario mural.

Figura 10: Uso de espacios de interacción

\section{Foro de discusión}

Es un espacio de intercambio de ideas y opiniones. El tutor plantea un tema de discusión y losalumnos participan incorporando sus aportes. El tutor anima este espacio, a partir de los aportes de los participantes y las orientaciones entregadas por el equipo pedagógico, articulando en él las lecturas contempladas en cada unidad. 
En cada una de las unidades se generó un foro de discusión, los tema planteados para los foros en orden de las unidades fueron: ¿Por qué enseñar geometría?, Construcciones geométricas con material concreto y el apoyo de las TICs, Enseñanza de la geometría en forma transversal.

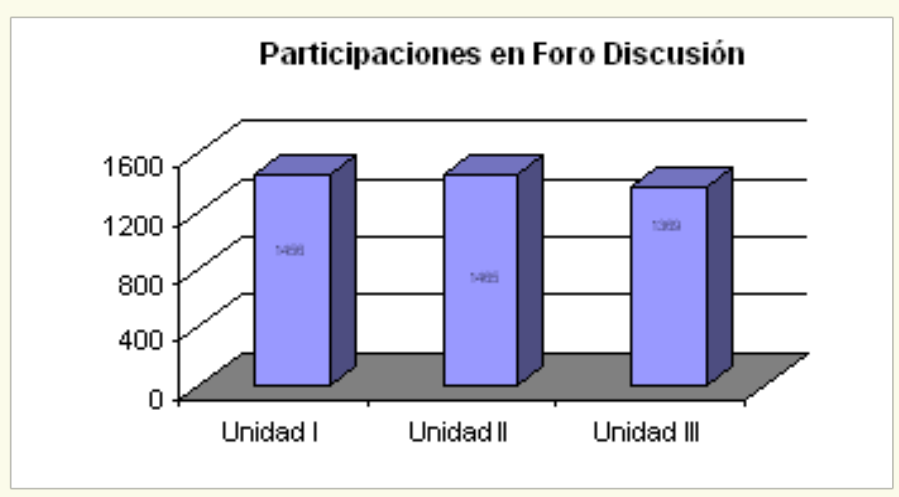

La figura 11 muestra la participación en los foros de cada unidad, en términos de resultados se observa una participación similar en los dos primeros foros, 1.456 en el foro de 1 unidad I y 1.465 en el de la unidad II, con un promedio de 50 intervenciones por foro en cada unidad.

Figura 11: Participación en foro de discusión

En la unidad III diminuye a 1.369, lo que se pude deber al número de deserciones y a la época del año, además que hacia el final del curso los alumnos privilegiaron los contenidos y el desarrollo de las actividades por sobre las intervenciones en los espacios interactivos. Las figuras 12 y 13 muestran un foro abierto por el tutor y la intervención posterior de un participante.

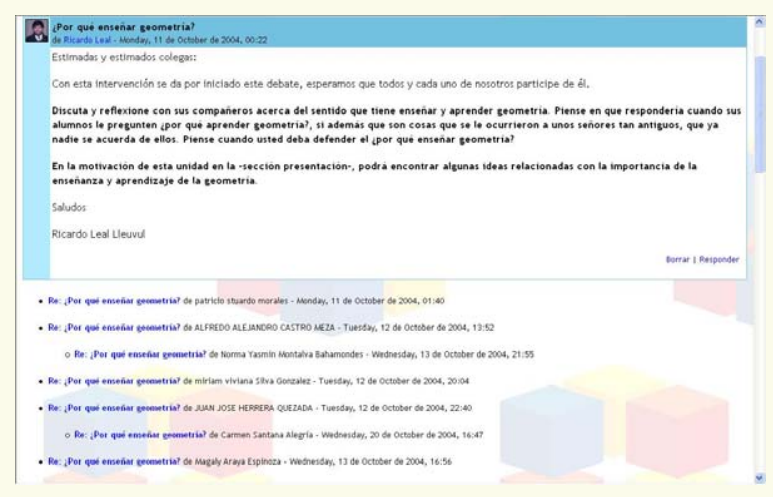

Figura 12: Foro discusión

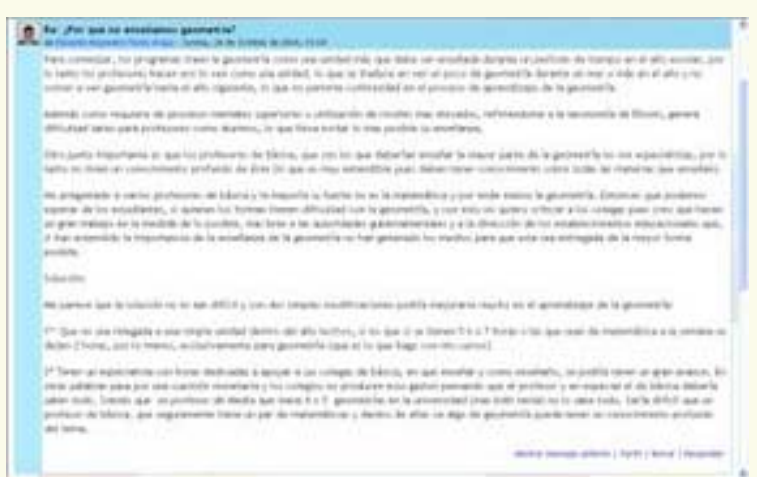

Figura 13: Intervención de un participante

\section{Consultas}

Consultas es un espacio donde los participantes pueden plantear, preguntas relacionadas con los contenidos, y las actividades. Recibiendo apoyo del tutor o compañeros más avanzados. El tutor puede derivar la consulta al equipo pedagógico. Para organizar la participación en este foro, es necesario indicar: semana y número de la actividad previo al titulo de la duda.

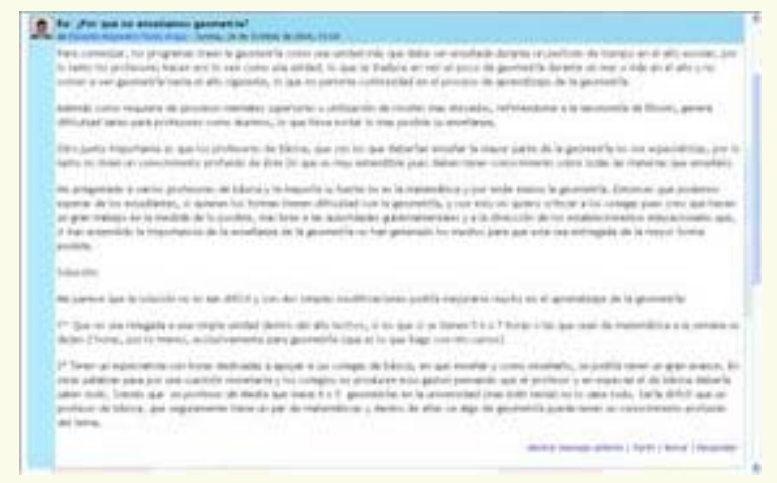

La participación en este espacio (figura 14) registra un total de 408 consultas realizadas por los participantes.

Se observa que del total de consultas 49.5\% (202) se realizaron en la primera unidad, un $29,7 \%$ en la unidad II y un $20,8 \%$ en la unidad III.

Figura 14: Participación en foro de discusión

Las figuras 15 y 16 muestra el espacio “consultas” y un ejemplo de una de ellas. 


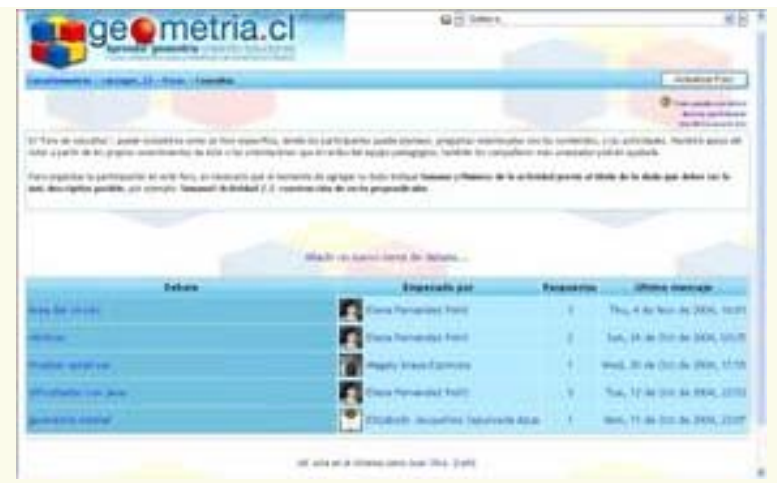

Figura 15: Foro “Consulta”

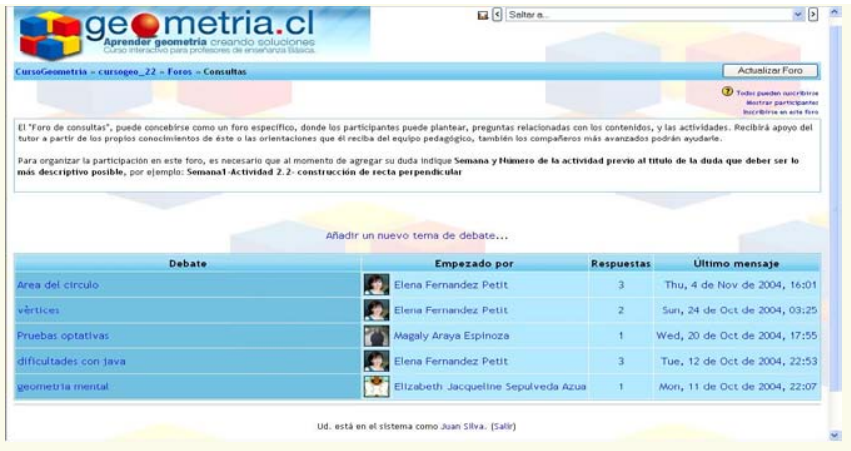

Figura 16: Consulta realizada por participante

\section{Diario Mural}

Es un espacio para compartir, recursos relacionados con los contenidos del curso: software, direcciones web, referencias de artículos o libros. Para organizar la participación en este espacio, se pide indicar: nombre del recurso los contenidos que se tratan y su posible uso pedagógico.

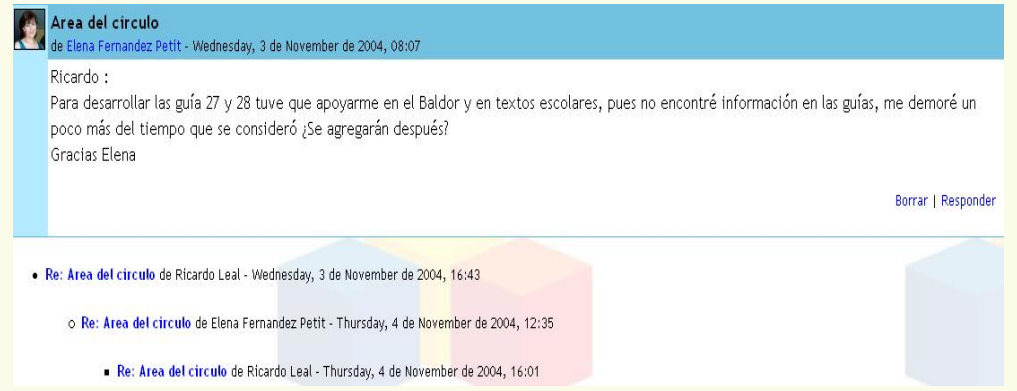

Figura 17: Participación en “Diario Mural”
La participación en este espacio (Figura 17) registra un total de 1.829 aportes al diario mural, realizadas por los participantes.

Se observa una distribución más homogénea concentrando las unidades I y III los mayores aportes con $37.4 \%$ y $34.3 \%$.

Las figuras 18 y 19 muestran un “Diario Mural” y un ejemplo de intervención de una participante.

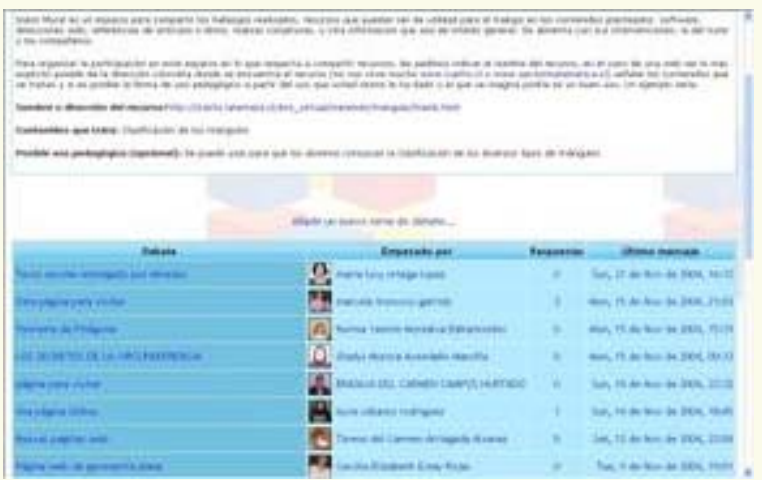

Figura 18: Foro “Diario Mural”

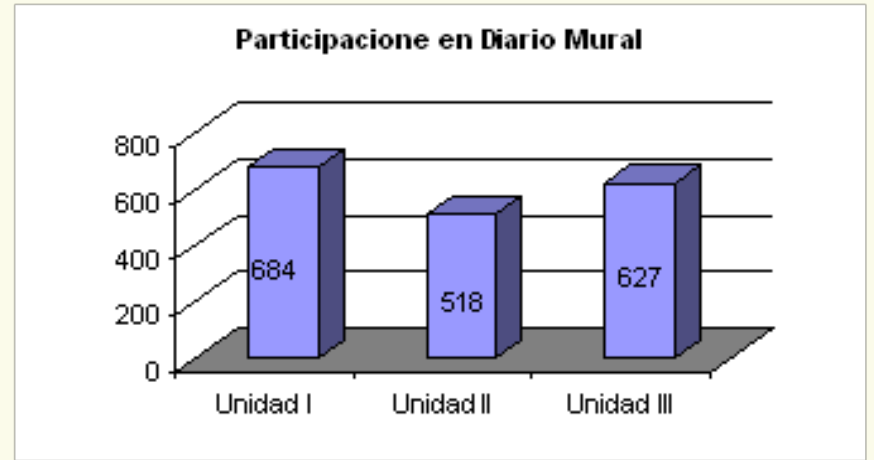

Figura 19: Participación en "Diario Mural”

\section{Evaluación}

La evaluación del curso contempla pretest, postest, evaluaciones sumativas por unidad; participación en los espacios interactivos, prueba final del curso, además de las evaluaciones formativas.

\section{Pretest y Postest}

Al inicio del curso se aplicó un Pretest respondido por 658 participantes, con un promedio de 4.3. Al finalizar se aplicó un Postest respondido por 645 participantes, alcanzándose un promedio de 5.8.

Se observa (Figura 20) que para el Pretest, el promedio decrece en la medida que las unidades hacen mención a tópicos menos conocidos y/o trabajados por los docentes. 


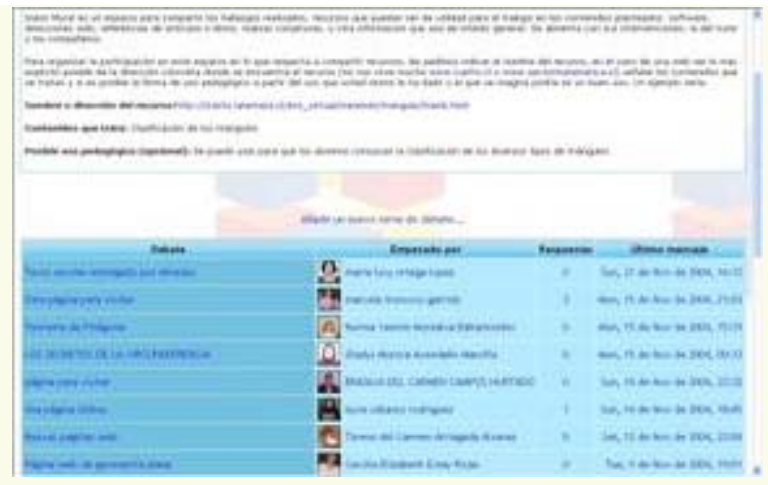

En el PostTest a nivel general y por unidad se notan avances significativos. A nivel general la diferencia es de 1.5 puntos y a nivel de unidad, 1.4 en la unidad I, 1.5 en la Unidad II y 2.0 en la Unidad III.

Figura 20: Resultados Pre y Post Test

\section{Evaluaciones Sumativas}

Cada unidad finaliza con una evaluación Sumativa compuesta por un conjunto de 18 preguntascon alternativas, el participante tiene 2 intentos para responderla, la nota final corresponde al promedio de los intentos, por cada intento se muestra la nota obtenida (escala de 1 a 7), hay una semana aproximadamente para responderla y luego de ella el participante puede revisar la prueba desplegándose sus respuestas y las correctas.

El promedio general de las evaluaciones sumativas alcanza a un 6.2, el promedio por unidades alcanzan a 6.6, 5.9 y 6.1 para las unidades I, II y III respectivamente.

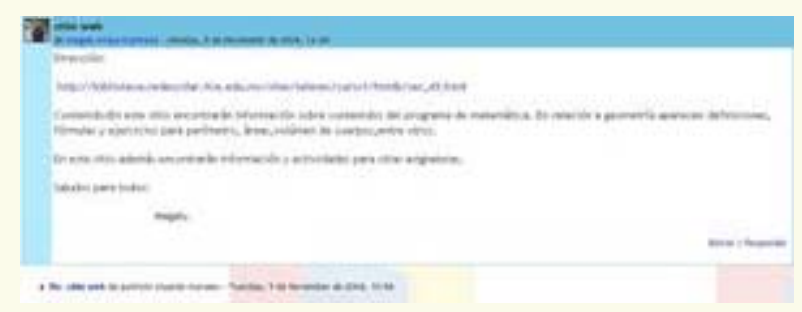

Figura 21: Resultados de la Evaluaciones
En las tres unidades se observa una ganancia respecto al Pretest que es significativa, 1.9 puntos para el caso de la Unidad 1 y 1.8 puntos en la Unidad II y 2.5 para la unidad III. Se observa el mismo fenómeno del postest en términos que la unidad con más avance es la unidad III. Todas las unidades están por debajo del PosTest.

Estas evaluaciones Sumativas fueron respondidas por 786 participantes en el caso de la Unidad I, 716 en el caso de la Unidad II y 646 para la Unidad III. Los que rinden la Evaluación III, alcanza a un 82\% de los que rinden la Evaluación I

\section{Evaluación Participación}

La participación en los espacios interactivos, es evaluada en cada unidad con los siguientes criterios: intervenciones a partir de las preguntas plateadas por el tutor; intervenciones que respondan a las intervenciones de sus compañeros; aportes al "Diario Mural". Cada uno de estos aspectos tienen igual incidencia en al evaluación de esta componente. El promedio alcanzado por evaluaciones en las interacciones alcanzó a un 5.3.

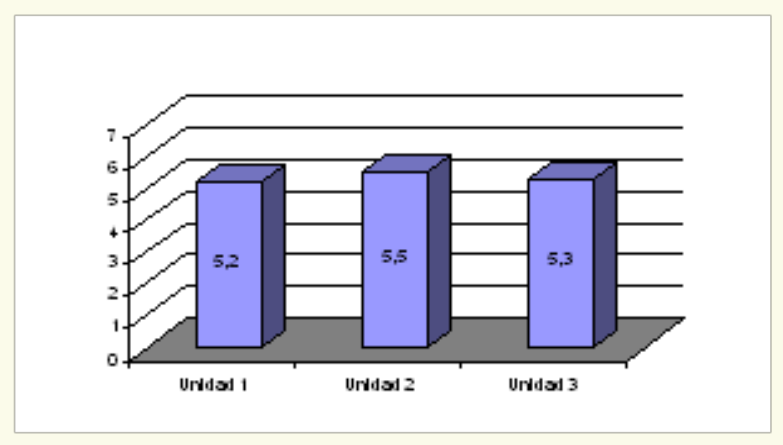

Se observa (Figura 22) un promedio relativamente uniforme entre las tres unidades. El promedio esta por debajo de las evaluaciones sumativas de la Unidad y también del PosTest. Esto es llamativo pues a pesar que la ponderación de la evaluación de las interacciones en la nota final dobla a la evaluaciones sumativas, los docentes le dieron menos importancia y no participaron masivamente.

Figura 22: Evaluación participación 
Un total de 675 participantes en la Unidad I, 574 en la Unidad II y 558 en la Unidad III, registraron interacción en alguno de los dos espacios. Se observa una disminución participando en la Unidad III un 17\% menos de los que lo hicieron en la Unidad I, Estos números también están por debajo de los que responden las evaluaciones sumativas en cada Unidad.

\section{Prueba Final}

La prueba final tiene una alto impacto en la nota final representa un $45 \%$ de ella, contiene 41 item, es decir es el post test más un complemento de 7 preguntas que buscaban medir los logros de los participantes en aspectos un poco más avanzados. La evaluación fue respondida por 645 participantes, el promedio final alcanzo a 5.7. Por unidades el promedio es 6.1 para la Unidad 1, 5.6 para la Unidad II y 5.5 para la unidad III.

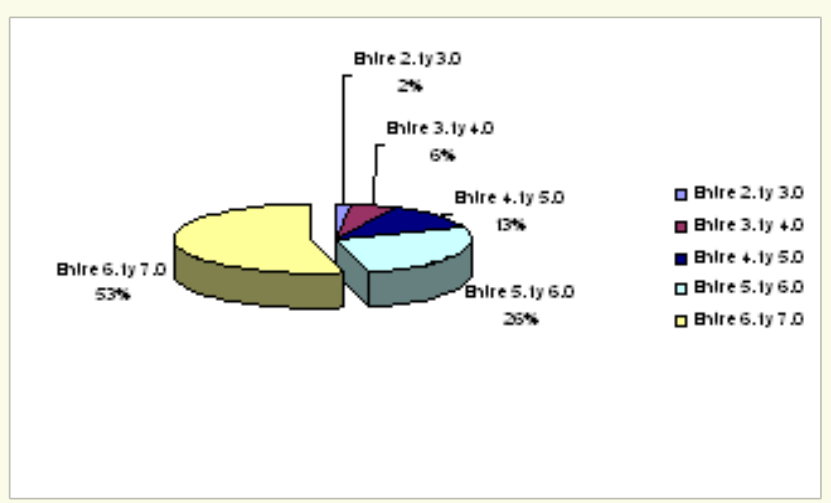

Figura 23: Resultados Prueba Final
Según la Figura 23, la mayor parte de los alumno un 54\% (348) alcanza nota entre 6.1 a 7 . La suma de alumnos que alcanza nota superior a 4.0 es un $82 \%$.

Adicionalmente se pude señalar que se registran 20 participantes que alcanzan la nota máxima 7.0.

Los resultados de esta evaluación no difieren significativamente a los mostrados anteriormente como resultados del postest, es decir que el nivel de logros en los item añadidos fue satisfactorio.

\section{Situación Final}

La evolución final considera las distintas evaluaciones formales las que se ponderan de la siguiente forma: evaluaciones finales de las unidades 15\%; presentación del portafolio 10\%; participación en espacios interactivos y diario mural $30 \%$; prueba final de Geometría -presencial- $45 \%$.

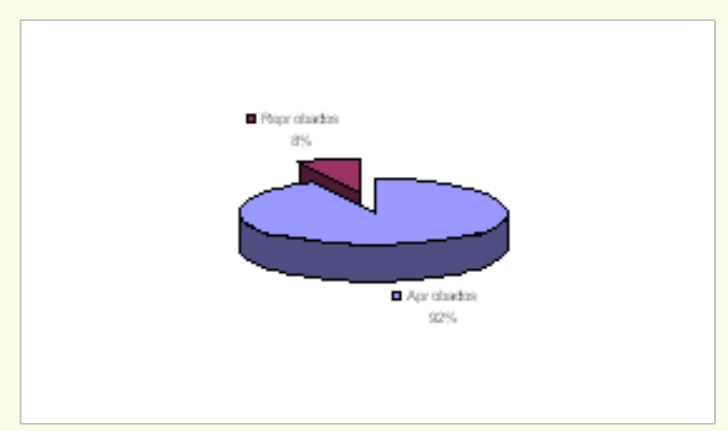

La figura 24 grafica muestra que del total de alumnos que finalizan el curso un 92\%(596) lo aprueba y un 8\%(49) lo reprueba.

La nota promedio de aprobación es un 5.5, existe

Figura 24: Situación Final

La nota mínima de aprobación fue cuatro (4.0, en escala de 1 a 7), obteniendo quienes lo aprueban una certificación del Centro Comenius de la Universidad de Santiago de Chile y CPEIP por 140 horas de perfeccionamiento

\section{Conclusiones}

El curso presentó un esfuerzo por entregarles a las profesores y profesoras de segundo ciclo de enseñanza primaria un proceso de perfeccionamiento de calidad, que permita construir los conocimientos, tanto disciplinares como didácticos, necesarios para que los participantes pueda mejorar sus prácticas pedagógicas. Lo anterior en una modalidad a distancia que favorezca la interacción con los compañeros y el tutor al interior de una comunidad de aprendizaje. Los principales conclusiones son: 
Alto interés por participar en el curso: El interés demostrado por los docentes para perfeccionarse en Geometría ha quedado plasmado en los altos números de inscritos y de matriculados, lo cual confirma la necesidad percibida de capacitar en esta área. Se registra un total de 1.004 participantes matriculados.

Alumnos activos: La cantidad de alumnos que se han mantenido activos en el curso es altamente positiva de los 1.004 inscritos originales 786 dieron la evaluación sumativa 1, un 78\% de participación efectiva, y entre éstos y los que rinden la evaluación final se produce un nivel de retención del 83\% de los participantes. Adicionalmente un promedio de 670 participantes se conectan semanalmente al curso, un 85\% de los participantes activos.

Valoración de los contenidos y recursos: Los contenidos del curso y los diversos recursos que este provee han sido valorados por los participantes, debido a su calidad, contextualización y la factibilidad que ellos los puedan usar y transferir al trabajo en el aula. Las aplicaciones Applets han sido dentro de este conjunto las más novedadosas, pues simulan construcciones geométricas.

Los encuentros presenciales Los aspectos positivos de los encuentro presenciales se centraron principalmente en la posibilidad de realizar trabajo colaborativo, compartir experiencias, aumentar la sensación de pertenencia y resolver dudas asociada a la metodología y a la utilización de la tecnología. La primera presencial presento problemas en su desarrollo debido a la convocatoria y a problemas con la plataforma, la segunda se desarrollo con normalidad. Los participantes han sugerido para próximas versiones incorporar trabajo relacionado directamente con los contenidos y algunos a pesar de ser un curso a distancia sugieren más presenciales.

La plataforma: La plataforma ha mostrado una gran estabilidad, sólo se vio con problemas en ciertos momentos puntuales en el desarrollo del curso, relacionados principalmente con los cuestionarios en línea, en términos generales ha estado en un alto porcentaje operativa y accesible. La forma en que se han dispuesto los espacios interactivos son evaluadas positivamente por los participantes. Destacan su facilidad de uso, la encuentran “amigable”, los espacios los usas con frecuencia y los encuentran útiles. En este sentido el proveer de espacios diferenciados para la discusión, el compartir recursos, aclarar dudas e interactuar en temas libres como el "foro social" creemos que es un elemento que contribuyen en aumentar la interacción y organizarla. Cuando a los participantes se les pregunta sobre la plataforma normalmente terminan hablando del curso y eso es una señal que se les hizo "invisible", se fundió en un solo gran elemento: el curso.

Las Interacciones: Se hizo un uso interesante por parte de los participantes de los espacios interactivos. Concentrándose las intervenciones en los foros de discusión 66\%, el "Diario mural" y "Consultas" registran un $28 \%$ y un $6 \%$ respectivamente de las intervenciones. Hubo también un espacio permanente en que el foro social que acaparó la mayor participación a partir de temas planteados por los participantes transformándose en una especie de "sala de profesores virtual". En este sentido creemos que la clave de la participación fue haber establecido espacios diferenciados para los tipos de intervenciones, que pudieron canalizar el tipo de intervenciones que los participantes normalmente realizan en estos cursos, ádemás de la animación del tutor especialmente en el foro de discusión.

Comunidad de tutores: La comunidad de tutores ha sido un espacio que ha permitido la coordinación del equipo pedagógico y tutorial que coordina el proyecto con los tutores, a través de ella se ha podido orientar y apoyar a los tutores en el desarrollo de su trabajo, los principales espacios utilizados han sido: orientaciones, consultas, solicitud de información y reportes, como se observa los dos primeros dedicados a lo pedagógico y los dos restantes a los administrativo. Se observa en esta comunidad un rol activo de los tutores, especialmente de los que logran mejores resultados en sus cursos.

Los tutores: Los tutores son agentes relevantes en el desarrollo del curso, ellos han desarrollado diversas tareas en las áreas: pedagógica, social, técnica y administrativa. El rol desempeñados por ellos especialmente al inicio del curso para "encantar" a los que no fueron a la presencial y en los tiempos de las evaluaciones para que los alumnos las rindan en los plazos establecidos ha sido vital para mantener a los alumnos activos. La labor de estos profesionales ha sido altamente valorada por los participantes, perciben en ellos un apoyo constante en el desarrollo del curso y sus actividades así como la aclaración de dudas de carácter pedagógico y administrativos, los perciben cercanos y siempre atentos a resolver sus dudas. Un factor que probablemente a contribuido son los reportes semanales que se les hizo llegar 
respecto a los participantes activos e inactivos de sus curso, esto les permiten determinar como va su curso en relación al sus pares a nivel nacional, varios de ellos han recibido las felicitaciones y reconocimiento del equipo pedagógico y de sus pares por los logros alcanzados.

Conformación de los grupos: En las regiones grandes como la Región Metropolitana donde se encuentra la capital del país, conformar los grupos de acuerdo al domicilio del docente, creemos que no es lo más optimo, pues traslada al ambiente virtual las divisiones que realizamos en el ámbito laboral. Profesores de establecimientos de comunas pobres con sus pares y los de establecimientos más acomodados con los suyos. Esto desde la perspectiva del construcción social de conocimiento y del concepto de Zona de Desarrollo Próximo de Vigostky no es muy adecuado. En este sentido creemos que la participación de docentes de establecimientos particulares puede convertirse en un aporte para el resto de la comunidad de aprendizaje, especialmente cuando se integran a grupos de sectores más populares.

Las Evaluaciones: Se observan a nivel general y de unidad, importantes avances en los aprendizajes reflejados en las diferencias de pre y post test. Adicionalmente las evaluaciones sumativas online también reflejan estos avances. Un elemento a nuestro juicio relevante es que la diferencia que se obtienen en relación a la pruebas sumativas online y el pre y post test reflejan que estas están significativamente más cercanas al post test, por lo cual dan cuenta de los aprendizajes adquiridos, superando la desconfianza inicial en términos que estas no reflejan los aprendizajes individuales toda vez que se presume culpable al docente de realizarla con apoyos adicionales a sus propios conocimientos.

El proceso seguido por los docentes participantes, ha sido en gran medida exitoso, sin duda perfectible en diversos aspectos. Ha significado el desarrollo de una experiencia virtual de formación docente que ha entregado a los participantes, una nueva forma de acceder a los contenidos, materiales de calidad e interacción con pares, tutor y especialistas, en una temática, prioritaria en la formación matemática de los niños Chilenos como lo es la geometría. La experiencia de este curso muestra un camino a seguir en estas nuevas formas de actualización docente que integran el uso de las TIC como un canal de comunicación y de formación durante la vida profesional, dando acceso a una experiencia formativa que muchos de los docentes participantes no hubiesen tenido acceso en los formatos tradicionales de formación presencial.

\section{Bibliografía}

ANDERSON, T \& KANUKA, H. (1997). On-Line Forums: New Platforms for Professional Development and Group Collaboration, Journal of Computer-Mediated Communication (JCMC) [en línea], 3 (3).Disponible en: http://www.ascusc.org/jcmc/vol3/issue3/anderson.html [2003, Octubre 31]

BARBERÀ, E Y BADIA, A. (2004) Educar con aulas virtuales: Orientaciones para la innovación en el proceso de enseñanza y aprendizaje, Madrid: A. Machado.

BATES, A.W. (1995). Technology open learning and distance education, London/NewYork: Routledge.

BERGE, Z.L. (1995). Facilitating Computer Conferencing: Recommendations From the Field. Educational Technology. 35(1), 22-30. Disponible en: http://www.emoderators.com/moderators/teach_online.html [2003, Agosto 18]

BRASLAVSKY, C. (1999). Bases, orientaciones y criterios para el diseño de programas de formación de profesores. Revista Interamericana de Educación. No 19, pp 13-50. Madrid-España: Organización de Estados Iberoamericanos para la Educación, la Ciencia y la Cultura (OEI). http://www.campusoei.org/oeivirt/rie19.htm [2003, Julio 22]

BULTRON, C. (2000). Nuevas tendencias en educación Informe mundial sobre la comunicación y la información 1999-2000, (pp. 51-67). Ediciones UNESCO/CINDOC

CABERO, J. (2001). La aplicación de las TIC: ¿esnobismo o necesidad educativa?, Red Digital [en línea], 1. Disponible en: http://reddigital.cnice.mecd.es/1/firmas/firmas_cabero_ind.html [2002, Diciembre 22]

COLLECT \& ENLACES (2004). Encuesta: Educación en la Sociedad de la Información. En Enlaces Estadísticas 2005 (pp 9-22), http://www.enlaces.cl/libro/encuesta.pdf [2005, Mayo 15] 
COLL, C. (2001). Constructivismo y educación: la concepción constructivista de la enseñanza y el aprendizaje. En: Coll, C., Palacios, J. y Marchesi, A. (comps.). Desarrollo Psicológico y Educación II. Psicología de la Educación (pp. 157-186). Madrid: Alianza.

CROOK, CH. (1998). Ordenadores y aprendizaje colaborativo. Madrid:Morata/MEC Ministerio de educación y cultura (Título original: Computer and the collaborative experience of learning, London: Routledge, 1994)

GREENING, T. (1998). Building the constructivist toolbox: an exploration of cognitive technologies, Educational Technology, 38(2), 23-35.

GROS, B. (2002). Constructivismo y diseños de entornos virtuales de aprendizaje, Revista de Educación, $328,225-247$.

GROS, B. y SILVA, J.(2005). La formación del profesorado como docentes en los espacios virtuales de aprendizaje, Revista Iberoamericana de Educación, Número 36(1), http://www.campusoei.org/revista/tec_edu32.htm

GRÜNBERG, J. (2002) REDOCENTE: una investigación sobre colaboración electrónica entre docentes de matemáticas y ciencias. Nuevas Tecnologías en Educación, Montevideo, Uruguay Universidad de la República. http://www.prc-antel.org.uy/nte/on-line/modulo_3.htm\#3 [2003, Diciembre 13]

HARASIM, L., HILTZ, S., TUROFF, M. \&TELES, L. (2000). Redes de aprendizaje: Guía para la enseñanza y el aprendizaje en red, Barcelona:Gedisa/EDIUOC [Versión original: Learning networks. A fiel guide to teaching and learning online. Cambridge (EE.UU.): Massachusetts Institute of Technology Press,1995)

HERNÁNDEZ, P. (1997). Construyendo el constructivismo: criterios para su fundamentación y aplicación escolar. En Rodrigo, $\mathrm{M}^{\mathrm{a}}$.J. Arnay, J. (Comps). La construcción del conocimiento escolar (pp. 285-312). Barcelona/Buenos Aires/México:Paidós

PÉREZ, A. (2002). Elementos para el análisis de la interacción educativa en los nuevos entornos de aprendizaje, pixel-bit revista de medios y educación [en línea], 19. Disponible en: http://www.sav.us.es/pixelbit/articulos/n19/n19art/art1904.htm [2003, Abril 1]

RYAN, S., SCOTT, B., FREEMAN, H. \& PATEL, D. (2000). The virtual university: the Internet and resoursce-based learning, London: Kogan Page.

SALMON, G. (2000). E-moderating: The key to teaching and learning online, London: Kogan Page.

SWAN, K., SHEA, P. FREDERICKSEN, E., PICKETT, A. PELZ, W., MAHER, G. (2000). Building knowledge building communities: consistency, contact and communication in virtual classroom, Journal Educational Computing Research, 23 (4), 359-381.

VYGOSTKY, L.S.(1978). El desarrollo de los procesos psicológicos superiores, Barcelona:Crítica.

TOLMIE, A. \& BOYLE, J. (2000). Factors influencing the success of computer mediated communication (CMC) environments in university teaching: a review and case study, Computers \& Education, 34 (2), 119-140.

\section{Notas:}

[1] La unidad de tiempo considerada es una hora cronológica (sesenta minutos). 


\title{
Lourdes Pérez Sánchez
}

Correos electrónicos por orden: fird@usal.es

\author{
o bien lopesan@usal.es
}

